\title{
Kepler Reliability and Occurrence Rates
}

\author{
Steve Bryson ${ }^{1}$ and The Kepler Team \\ ${ }^{1}$ NASA Ames Research Center email: steve.bryson@nasa.gov
}

The Kepler mission has produced tables of exoplanet candidates ("KOI table"), as well as tables of transit detections ("TCE table"), hosted at the Exoplanet Archive (http://exoplanetarchive.ipac.caltech.edu). Transit detections in the TCE table that are plausibly due to a transiting object are selected for inclusion in the KOI table. KOI table entries that have not been identified as false positives (FPs) or false alarms (FAs) are classified as planet candidates (PCs, Mullally et al. 2015). A subset of PCs have been confirmed as planetary transits with greater than $99 \%$ probability, but most PCs have < 99\% probability of being true planets. The fraction of PCs that are true transiting planets is the $\mathrm{PC}$ reliability rate. The overall $\mathrm{PC}$ population is believed to have a reliability rate $>90 \%$ (Morton \& Johnson 2011).

The PC reliability rate is not homogeneous across the PC population: low $\mathrm{S} / \mathrm{N}$ transiting objects, particularly Earth-size planets at 365-day orbital periods, may have reliability rates substantially lower than $90 \%$. Astrophysical false positives, such as grazing or background eclipsing binaries, are identified through pixel analysis that determines the location of the transit source, or through light curve shape analysis that determines the nature of the transiting object. When the transit signal is very shallow both pixel and light curve analysis can fail, resulting in a detected transit that cannot be localized to the target star and whose nature cannot be determined. Such objects are classified as planet candidates, but have a lower reliability than higher $\mathrm{S} / \mathrm{N}$ objects that can be localized and whose light curve shapes can be analyzed.

A variety of instrumental phenomena can generate spurious detections not caused by a transiting object. Such FAs can be very difficult or impossible to distinguish from shallow planetary transits. A particularly important class of FAs are thermal systematics that lead to a large excess of shallow transit detections near the Kepler 372-day orbital period. Other FA sources include temporary pixel sensitivity changes due to cosmic rays, and stellar variability.

Exoplanet occurrence rate calculations, particularly in the $\eta_{\oplus}$ regime, require understanding the rate of the above FPs and FAs relative to the rate of true planets in the PC population. Studies by the Kepler mission are currently underway that should significantly improve our understanding of reliability. These studies include transit searches of inverted and shuffled data. Real planets will not be detected in either study, so they will provide insight into FA distributions.

Once our understanding of reliability has improved, occurrence rate estimates such as that in Burke et al. (2015) will need to be modified to use that understanding. The best way to use knowledge of PC reliability in occurrence rate calculations is currently an open problem.

\section{References}

Burke, C. J., et al. 2015 ApJ 809, 1

Morton, T. D. \& Johnson, J. A. 2011, ApJ 738, 170

Mullally, F., et al. 2015, ApJS 21,1 31 